\title{
$\mathrm{CH}$ \\ Intramolecular Cyclopropanation of 1,4-Dienes through Hydroboration-Homologation: Easy Access to Bicyclo[3.1.0]hexanes
}

G. Xu and P. Renaud*, Angew. Chem. Int. Ed. 2016, 55, 3657. University of Bern

Bicyclo[3.1.0]hexanes are present in many natural and non-natural products. Due to their ring strain, they are also interesting synthetic intermediates in ring-opening reactions. Yet, the same ring strain makes their synthesis difficult and challenging. Xu and Renaud now present an uncatalyzed and spontaneous intramolecular cyclopropanation process involving B-(1-chloroalkyl)catecholborane intermediates coming from selective hydroboration of dienes and subsequent Matteson homologation. A mechanistic study suggests that the reaction proceeds via unexpected carbocationic intermediates. The cascade procedure involves the formation of three new sigma $\mathrm{C}-\mathrm{C}$ bonds at the same carbon atom and it can be applied to the synthesis of various bicyclo[3.1.0]hexanes.

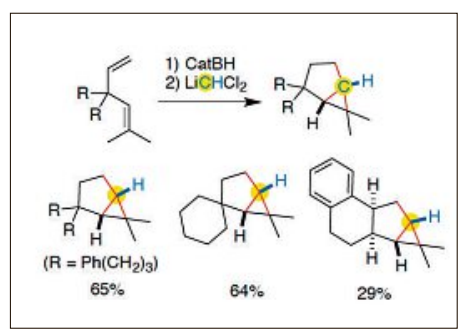

\section{Direct Visualization of Excited-State Symmetry Breaking Using Ultrafast Time-Resolved Infrared Spectroscopy}

B. Dereka, A. Rosspeintner, Z. Li, R. Liska, and E. Vauthey*, J. Am. Chem. Soc. 2016, 138, 4643. University of Geneva

Fluorescence spectra of symmetric multibranched donor-acceptor (DA) molecules exhibit a strong solvatochromism indicative of a dipolar $S_{1}$ state. This striking phenomenon has been explained by symmetry breaking in the excited state. Using femtosecond time-resolved infrared spectroscopy, Vauthey and coworkers monitor the symmetry-breaking dynamics of the electronic distribution upon photoexcitation of a quadrupolar D- $\pi$-A$\pi$-D molecule. The solvent plays a pivotal role in both the extent of the symmetry breaking and its dynamics. Therefore, the nature of the emissive state can be tuned from quadrupolar to dipolar by choice of the solvent. This process should be universal for symmetric molecular architectures with multipolar electronic distribution, from relatively small molecules to larger systems, such as dendrimers and polymers.

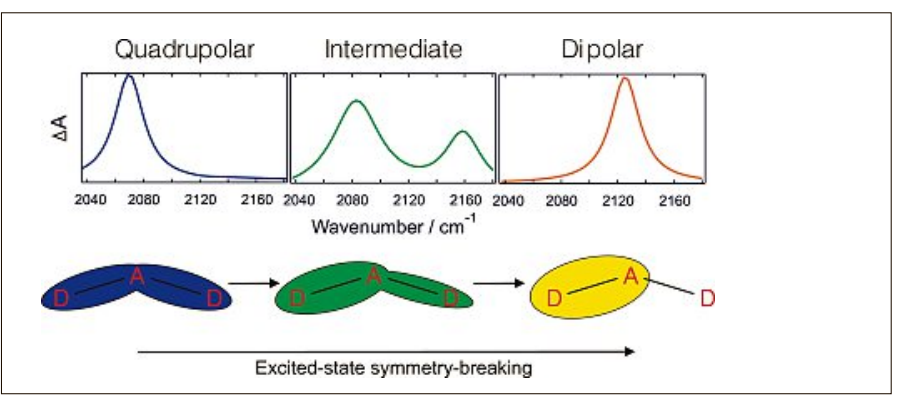

\section{Novel Chemoselective ${ }^{18} \mathrm{~F}$-Radiolabeling of Thiol- Containing Biomolecules under Mild Aqueous Conditions}

A. Chiotellis, F. Sladojevich, L. Mu, A. Müller Herde, I. E. Valverde, V. Tolmachev, R. Schibli, S. M. Ametamey, and T. L. Mindt*, Chem. Commun. 2016, 52, 6083. ETHZürich, University of Basel

The selective functionalization of peptides and proteins with molecular imaging probes (e.g., radionuclides) commonly involves cysteine-specific conjugation chemistry using maleimides. However, ${ }^{18} \mathrm{~F}$-labeled maleimide reagents require multistep synthesis and the Michael-type addition products formed by the reaction with thiols lack defined stereochemistry and sufficient stability in vivo. Mindt and collaborators now report the onestep preparation of a novel ${ }^{18} \mathrm{~F}$-labeled heterocyclic methylsulfone and its utility as a prosthetic group for the rapid, stable, and chemoselective conjugation with thiol-containing (bio)molecules under aqueous conditions. The new methodology was applied successfully to the ${ }^{18} \mathrm{~F}$-labeling of tumor-targeting peptides and affibody proteins.
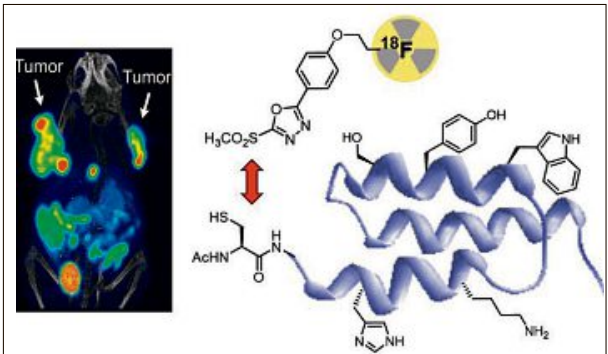

\section{Synthesis of Quaternary $\alpha$-Perfluoroalkyl Lactams via Electrophilic Perfluoroalkylation}

D. Katayev, J. Václavík, F. Brüning, B. Commare, and A. Togni*, Chem. Commun. 2016, 52, 4049. ETH Zürich

The presence of fluorine atoms in an organic molecule may have profound effects on the molecule's biological properties. Not surprisingly, the selective introduction of fluorine atoms into drug or other molecules is a topic of great interest. Togni and coworkers developed an efficient method for the facile $\alpha$-fluoroalkylation of lactam-derived ketene silyl amides by hypervalent iodine reagents under trimethylsilyl triflimide catalysis. The simplicity, efficiency and the broad scope of this protocol bears potential for applications in medicinal chemistry, agrochemistry and the material sciences.

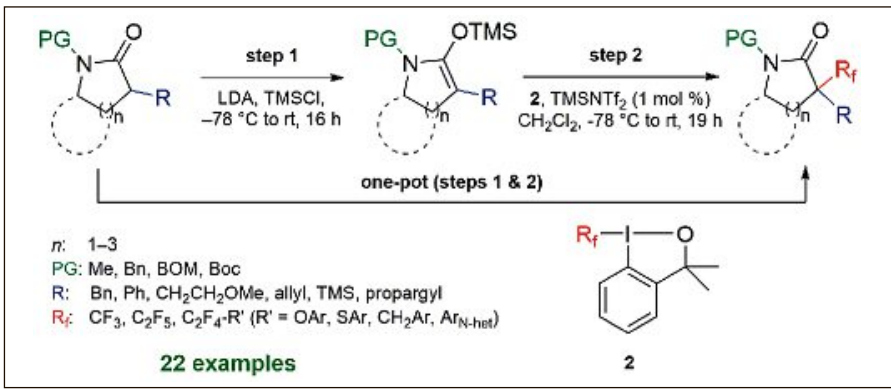

Prepared by Caroline D. Bösch, Markus Probst, Yuliia Vyborna, Mykhailo Vybornyi, Simon M. Langenegger, and Robert Häner* Do you want your article to appear in the SWISS SCIENCE CONCENTRATES highlight?

Please contact robert.haener@dcb.unibe.ch 